\title{
MHD ACCRETION IN A BLACK HOLE MAGNETOSPHERE
}

\author{
M. TAKAHASHI \\ Department of Physics and Astronomy, Aichi University of \\ Education \\ Hirosawa, Igaya-cho, Kariya 448, Japan
}

\section{Introduction}

To explain the activity of active galactic nuclei or compact X-ray sources, I consider a black hole magnetosphere in the center of these objects. The considering black hole magnetosphere is composed of a massive black hole with surrounding fluids and magnetic fields, and rotates rapidly. Because of the strong gravitation and the rapid rotation, both an accretion and a wind/jet would be generated from plasma sources (e.g., an accretion disk and its corona). The outgoing flow carries the angular momentum from the plasma source effectively, and then the accretion would go on stationary, releasing its gravitational energy. I assume that the magnetosphere is stationary and axisymmetric, and that the ideal MHD approximation is available for the streaming fluid. I discuss the thermal effects on MHD flows, and then I argue that the trans-fast MHD accretion solution can be broken by highly thermal effects.

\section{Trans-magnetosonic accretion}

To understand flow properties in the black hole magnetosphere, we solve the relativistic Bernoulli equation (Camenzind,1986; Takahashiet al.,1990), which has a form of $d\left(\ln u^{r}\right) / d r=N\left(r, u^{r}\right) / D\left(r, u^{r}\right)$, with a radial magnetic field line, where $u^{r}$ is the radial four-velocity of the fluid. To accrete onto the black hole or blow away to far distant region, the MHD flow ejected from plasma sources with low-velocity must pass through the slow magnetosonic point, the Alfvén point and the fast magnetosonic point in order, where we see that $D=0$ and we must require $N=0$ at the same point to get a physical solution. These 'critical conditions' restrict the MHD flow's five parameters (see Bondi,1952; Weber \& Davis,1967; Camenzind,1987). So, I 
(a)

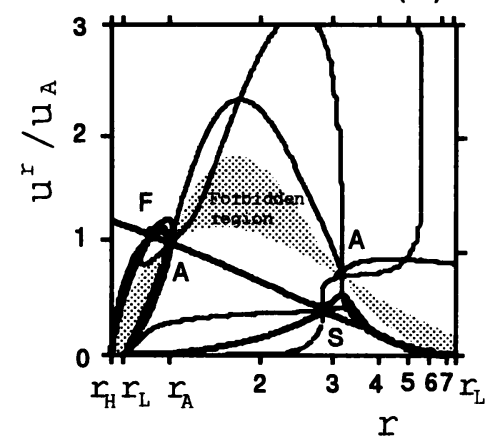

(b)

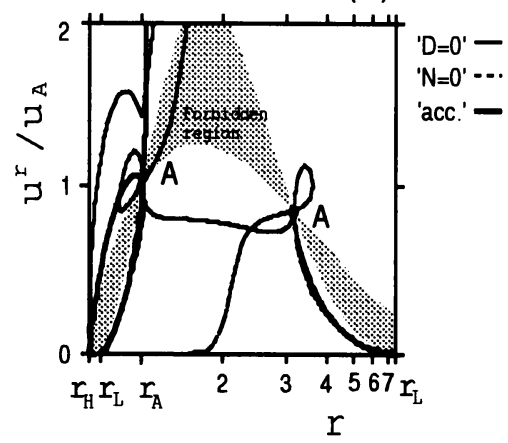

Figure 1. MHD accretion on to the black hole $(a / m=0.1)$. [a] shows the case of a strong magnetic field $\left(C_{s}\right)_{\mathrm{A}}<u_{\mathrm{A}}$; and [b] shows the case of a weak magnetic field $\left(C_{s}\right)_{\mathrm{A}}>u_{\mathrm{A}}$. For the case of $\left(C_{s}\right)_{\mathrm{A}}<u_{\mathrm{A}}$, we always find the crossing of $N=0$ line and $D=0$ line between the Alfvén point and the event horizon, while for the case of $\left(C_{s}\right)_{\mathrm{A}}>u_{\mathrm{A}}$ the crossing is limited.

can evaluate the energy flux of the trans-fast MHD flows as functions of the fast magnetosonic radius, the Alfven radius, the light radius and the location of plasma injection point (Takahashi, 1994).

When the sound four-velocity $C_{s}$ is greater than the Alfven wave speed at the Alfvén radius $u_{\mathrm{A}}$, we find a restriction by the thermal effect onto the hot trans-fast MHD accretion. In this case, it is possible that no physical trans-fast MHD accretion solution exists between the Alfvén radius and the event horizon. We only obtain unphysical solutions that at the event horizon the plasma's pressure diverges and its radial velocity $\left(u^{r}\right)_{\mathrm{H}}$ becomes zero. This means that an stationary and axisymmetric ideal MHD accretion onto black hole do not happen along such a magnetic flux tube. To realize a physical accretion flow, the configuration of magnetic field lines would be changed by the thermal-pressure dominated flow. If a shock front is generated at somewhere between the plasma source and the event horizon, the trans-fast MHD accretion would be realized with different wind parameter sets.

\section{References}

Bondi,H. 1952, Mon. Not. R. Astron. Soc., 112,195

Camenzind,M. 1986, Astron. Astrophys., 162, 32

Camenzind,M. 1987, Astron. Astrophys., 184, 341

Takahashi,M., Nitta,S., Tatematsu,Y., \& Tomimatsu,A. 1990, Astrophys. J., 363, 206

Takahashi,M. 1994, in Proceedings of the Fourth Workshop on

General Relativity and Gravitation, p24, ed. K.Maeda

Weber,E. J., Davis,L.,Jr. 1967, Astrophys. J., 148, 217 